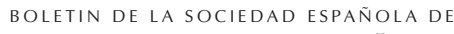

\section{Microestructura y propiedades de materiales cerámicos PZT con control de crecimiento de grano}

\author{
L. A. CELI, A. C. CABAllero, M. VilleGAS, P. DURÁN, C. MOURE, J. F. FERNÁNDEZ. \\ Departamento de Electrocerámica, Instituto de Cerámica y Vidrio, CSIC. 28500 Arganda del Rey, Madrid.
}

\begin{abstract}
Se ha realizado un proceso de modificación superficial con ester fosfato al $0.3 \%$ en volumen, sobre polvo cerámico de titanato circonato de plomo, PZT. Se observa que el material modificado con fósforo presenta una mayor densificación aparente a una temperatura menor unida a una reducción de las pérdidas de peso durante el proceso de densificación. Se han establecido las relaciones entre el procesamiento y las microestructuras del material PZT sin modificar y el modificado. Se evidencia un control del crecimiento de grano y una mayor homogeneidad en la distribución de tamaños de grano en el material modificado.

Palabras clave: Titanato Circonato de Plomo (PZT), microestructura, sinterización, control de crecimiento de grano.

Microstructure and properties of PZT ceramics with grain growth control

Lead zirconate titanate ceramic powders have been surface modified by using phosphor esther $0.3 \%$ in volume. The phosphorous modification gave higher densities at lower temperatures associated with a reduction of the weight losses during the densification process. From the relationships between ceramic processing and microstructure, it was established that the phosphorous surface modification allows the effective grain growth control as well a higher homogeneity in the grain size distribution.
\end{abstract}

Key words: Lead zirconate titanate (PZT), microstructure, sintering, grain growth control.

\section{INTRODUCCIÓN.}

Los materiales cerámicos de titanato circonato de plomo, PZT, han sido utilizados como materiales piezoeléctricos en una gran variedad de aplicaciones (1-2), debido a la versatilidad de adaptación de sus propiedades a las demandas del mercado. Sin embargo, estos materiales cerámicos presentan algunos inconvenientes en el proceso de fabricación que redundan en su elevado costo de producción. Entre estos inconvenientes se pueden encontrar variaciones en las propiedades del material cerámico PZT relacionadas, en su caso, a pérdida de estequiometría, porosidad, inhomogeneidad en el tamaño de grano, existencia de fases secundarias y diferencias locales en la polarización. Uno de los aspectos a mejorar está asociado a lograr un mayor control de la microestructura del material (3-4). Debe evitarse crecimiento de grano no uniforme si se desean obtener materiales con propiedades dieléctricas y piezoeléctricas óptimas. El tamaño de grano ejerce una fuerte influencia sobre las propiedades elásticas, dieléctricas y piezoeléctricas de cerámicas de PZT (5-8). La modificación superficial de las partículas del polvo cerámico $\mathrm{BaTiO}_{3}$ con el dispersante orgánico ester fosfato, empleando un proceso de turbinado a alta velocidad, ha mostrado excelentes resultados, obteniéndose materiales con microestructura homogénea, y una fuerte inhibición del crecimiento de grano (9-10). A diferencia de los métodos tradicionales de dopado, este método de modificación superficial permite una distribución uniforme del dopante sobre las partículas del polvo cerámico (9) produciendo un mejor comportamiento durante los primeros estadios de la sinterización. El origen de este comportamiento se halla en la interacción que se produce entre el fósforo y el bario, formando una fase reactiva que recubre los granos y controla el proceso de sinterización en su primera etapa (1112). Las características del catión $\mathrm{Pb}^{2+}$ permiten suponer "a priori" una interacción con el fósforo similar a la que ocurre con el bario, y por lo tanto, parece posible obtener con el mismo procedimiento que el empleado para el $\mathrm{BaTiO}_{3}$, materiales de PZT con microestructura controlada.

Por tanto el objetivo de este trabajo es observar el resultado de la modificación superficial de las partículas del polvo cerámico PZT sobre la microestructura de este material, y por consiguiente sobre sus propiedades finales. Estos resultados se comparan con los del material PZT no modificado.

\section{PROCEDIMIENTO EXPERIMENTAL}

Se ha partido de polvo cerámico PZT dopado con $\mathrm{Nb}$ en $1.5 \%$ at., obtenido previamente (13), mediante el método de calcinación reactiva (14) y de composición en la frontera morfotrópica de fases, $\mathrm{Zr} / \mathrm{Ti}=53 / 47$. El tamaño promedio de partícula de este polvo cerámico es $0.37 \mu \mathrm{m}$ y la superficie específica de $1.7 \mathrm{~m}^{2} / \mathrm{g}$. La curva de distribución de tamaño de poros abarca poros de tamaño $\sim 70 \mathrm{~nm}$ hasta 9 micras, y el pico de máxima intrusión de mercurio está centrado en $\sim 0.3 \mu \mathrm{m}$. El dispersante orgánico ester fosfato está compuesto del $60 \%$ de $\mathrm{C}_{4} \mathrm{H}_{11} \mathrm{O}_{4} \mathrm{P}$ y el $40 \%$ de $\mathrm{C}_{8} \mathrm{H}_{19} \mathrm{O}_{4} \mathrm{P}$ y tiene una densidad de 1.13 $\mathrm{g} / \mathrm{cm}^{3}$. El polvo cerámico PZT se dispersó en medio isopropí- 
lico con ester fosfato al $0.3 \%$ en volumen, mediante turbinado a alta velocidad de $10.000 \mathrm{rpm}$ durante 10 minutos. La mezcla se secó luego en una estufa a $60^{\circ} \mathrm{C}$ y se tamizó a $100 \mu \mathrm{m}$. Suponiendo que todo el fósforo del ester fosfato queda como $\mathrm{P}_{2} \mathrm{O}_{5}$, la cantidad de $\mathrm{P}_{2} \mathrm{O}_{5}$ añadida representa el $0.016 \%$ en peso de PZT. A este polvo cerámico se le denominó PZTF. Puesto que el proceso de turbinado afecta a las características del polvo cerámico (13) al romper aglomerados y variar el contenido de porosidad, el comportamiento de sinterización del material se verá alterado (15-16). Por tanto los efectos relacionados con la modificación del fósforo pueden ser encubiertos por el proceso de turbinado. En consecuencia, polvo cerámico PZT sin fósforo se turbinó con el fin de poder establecer comparaciones entre los dos polvos cerámicos e identificar directamente los efectos relacionados con la adición de fósforo.

Se añadió aglomerante orgánico al polvo cerámico y se mezcló homogéneamente en acetona, a fin de optimizar el proceso de prensado. La mezcla se secó en una estufa a una temperatura de $60^{\circ} \mathrm{C}$ durante algunos minutos. Luego se tamizó en un tamiz de $300 \mu \mathrm{m}$ y se prensó uniaxialmente bajo una presión de $\sim 45 \mathrm{MPa}$, en discos de $15 \mathrm{~mm}$ de diámetro y $1.5 \mathrm{~mm}$ de espesor. Los discos prensados fueron calentados hasta $600^{\circ} \mathrm{C}$ durante una hora a fin de eliminar el material orgánico. En el caso de las muestras modificadas con fósforo, este tratamiento térmico permitió la eliminación adicional de las cadenas orgánicas de ester fosfato, dejando únicamente el fósforo como residuo adsorbido en las superficies de las partículas del polvo cerámico PZT. Por consiguiente, las pérdidas de peso que presenten las muestras de PZT y de PZT modificado con fósforo, durante el proceso de sinterización, estarán relacionadas fundamentalmente con pérdidas de $\mathrm{PbO}$ por volatilización y por incorporación del $\mathrm{Nb}^{5+}$ en la red cristalina. Los discos prensados y tratados térmicamente se sinterizaron en un crisol sellado con $\mathrm{ZrO}_{2}$, dentro del cual se mantuvo una atmósfera de $\mathrm{PbO}$ creada por un tampón consistente en una mezcla $\mathrm{PbZrO}_{3}$ $+5 \% \mathrm{ZrO}_{2}$, a fin de controlar las pérdidas de peso por volatilización de $\mathrm{PbO}$. La sinterización se realizó a diferentes temperaturas y durante 2 horas, con una velocidad de calentamiento y de enfriamiento de $3^{\circ} \mathrm{C} / \mathrm{min}$. La densidad de los discos sinterizados se determinó mediante el método de Arquímedes, siendo el medio líquido el agua. Las pérdidas de peso se determinaron por diferencias de peso entre el material en verde y el

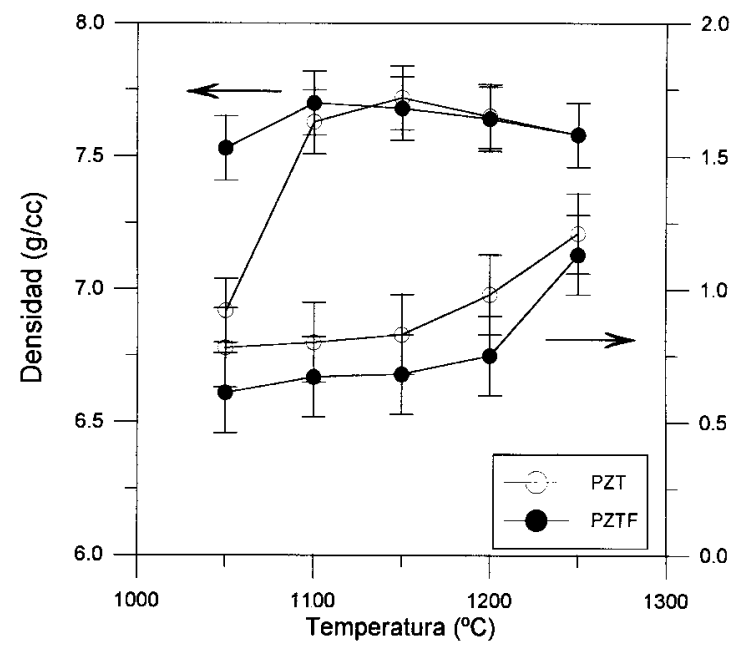

$\widehat{0}$
0
0
0
0
0
0
$\frac{0}{0}$
$\frac{0}{0}$
$\frac{0}{0}$
0
0

Figura 1. Densificación y pérdida de peso en función de la temperatura. material sinterizado. La microestructura se observó por Microscopía Electrónica de Barrido (MEB) sobre la superficie de las muestras sinterizadas. La distribución y el tamaño de grano se determinó por medio de un analizador de imágenes de Princeton Gamma-Tech, Inc. (programa IMAGIST II). Este programa determina el tamaño promedio del diámetro de la superficie proyectada en un plano, por lo cual se le hizo la corrección del factor de forma de 1.5 para partícula esféricas. La distribución del tamaño de grano se obtuvo mediante histogramas representados con 16 barras a fin de tener pequeños pasos de incremento de las barras. Estos histogramas se representaron mediante ajuste polinómico, cuya altura se normalizó con la intensidad máxima del respectivo histograma. La caracterización dieléctrica y piezoeléctrica se realizó sobre los discos desvastados hasta alcanzar un grado plano-paralelismo inferior a $20 \mu \mathrm{m}$ y electrodados con pintura de plata. El electrodo se sinterizó a $700^{\circ} \mathrm{C} 30$ minutos. El proceso de polarización se realizó bajo un campo de $40 \mathrm{kV} / \mathrm{cm}$, a una temperatura de $120^{\circ} \mathrm{C}$ durante 30 minutos. La determinación de las pro-
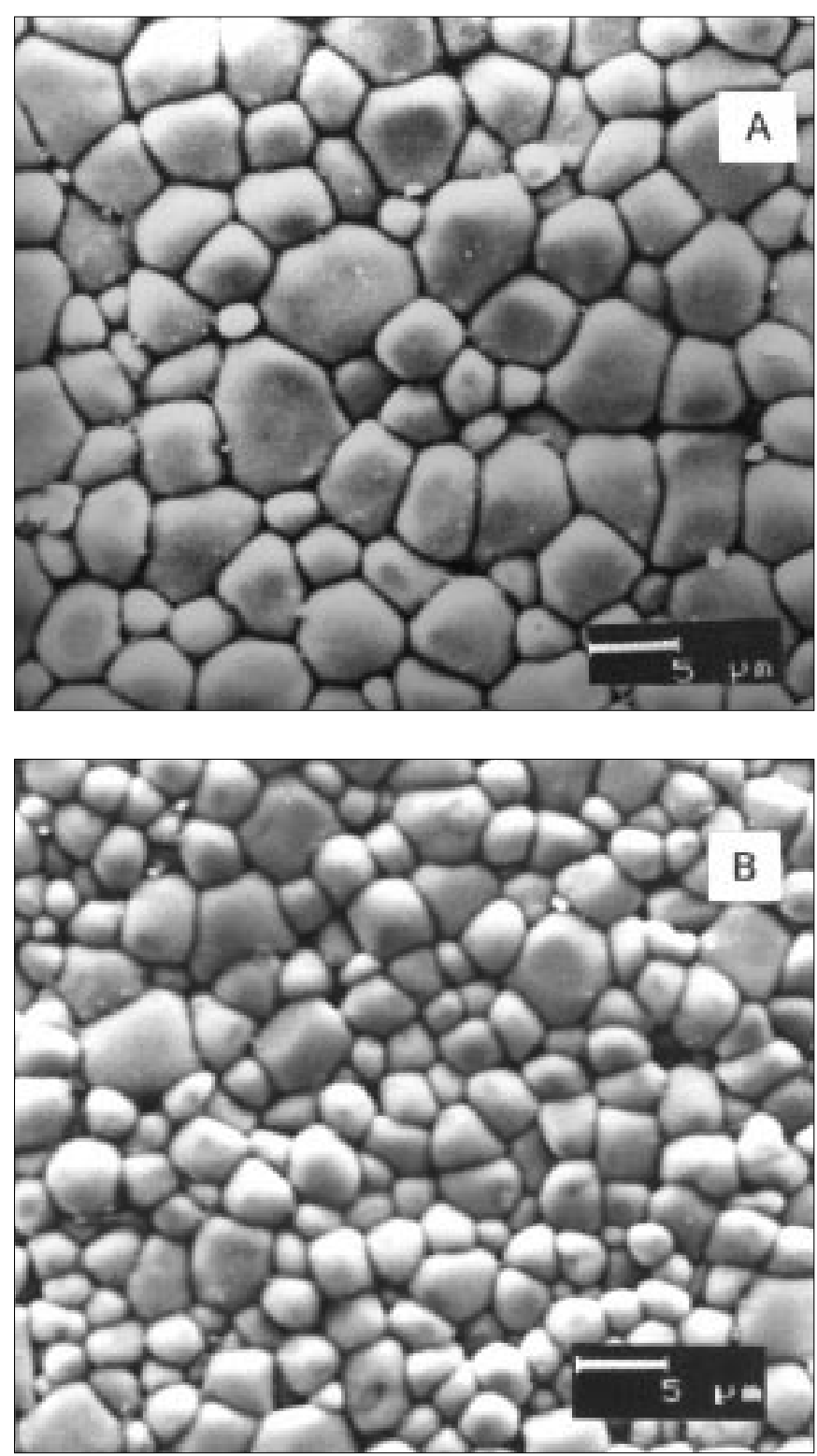

Figura 2. Micrografías MEB de materiales cerámicos sinterizados a $1200^{\circ} \mathrm{C} / 2 \mathrm{~h} \mathrm{A)} \mathrm{PZT,} \mathrm{B)} \mathrm{PZTF.}$ 
piedades dieléctricas y piezoeléctricas se realizó mediante un analizador vectorial de impedancias HP 4192 A, controlado por un ordenador HP 9216. Las propiedades piezoeléctricas se evaluaron de acuerdo con las normas IEEE (17). La determinación del parámetro $d_{33}$ se realizó en un equipo Berlincourt Piezo-d meter.

\section{RESULTADOS Y DISCUSIÓN}

La figura 1 muestra las curvas de densidad aparente en función de la temperatura de sinterización para ambos materiales. Las muestras de PZTF alcanzan valores de densidad aparente cercanos a $\sim 94 \% \delta_{\mathrm{t}}$ a temperaturas de sinterización de $1050^{\circ} \mathrm{C}$. Estos valores de densidad se alcanzan en las muestras de PZT a temperaturas próximas a $1100^{\circ} \mathrm{C}$. Ambos materiales cerámicos presentan valores elevados de densidad aparente ( $~ 96.5 \%$ $\delta_{\mathrm{t}}$ ) en el intervalo de temperatura de $1100^{\circ} \mathrm{C}-1150^{\circ} \mathrm{C}$. A partir de $1150^{\circ} \mathrm{C}$ las curvas de densidad aparente de ambos materiales cerámicos son casi coincidentes, presentando un comportamiento similar de pérdida de densidad.

Las curvas de pérdida de peso (figura 2) de ambos materiales presentan una tendencia similar, si bien las muestras de PZTF presentan una pérdida de peso inferior en $\sim 0.15 \%$ en peso, que las muestras de PZT en el rango de temperaturas en estudio. Las pérdidas de peso de las muestras PZT y PZTF indicadas en la figura 2 son productos de varios procesos. El material PZT experimenta una pérdida de peso durante el tratamiento térmico de sinterización debido a los mecanismos de incorporación de carga generados por la incorporación del catión $\mathrm{Nb}^{5+}$ en posiciones $\mathrm{B}$ de la red cristalina con estructura perovskita (18), y que se traducen en la eliminación del $0.48 \%$ en peso de $\mathrm{PbO}$ para el contenido de las muestras en estudio. Además, el análisis termogravimétrico (ATG-TG) de muestras previamente prensadas y quemadas a $600^{\circ} \mathrm{C} / 1 \mathrm{~h}$, presenta una pérdida de peso de $\sim 0.07 \%$ debido a eliminación de restos orgánicos y especies adsorbidas. Por tanto, todas las muestras de PZT y PZTF presentarían una pérdida de peso de $\sim 0.55 \%$ que debe ser restada de las pérdidas de peso que exhiben estas muestras en la figura 2. De esta forma, a $1050^{\circ} \mathrm{C}$, el material

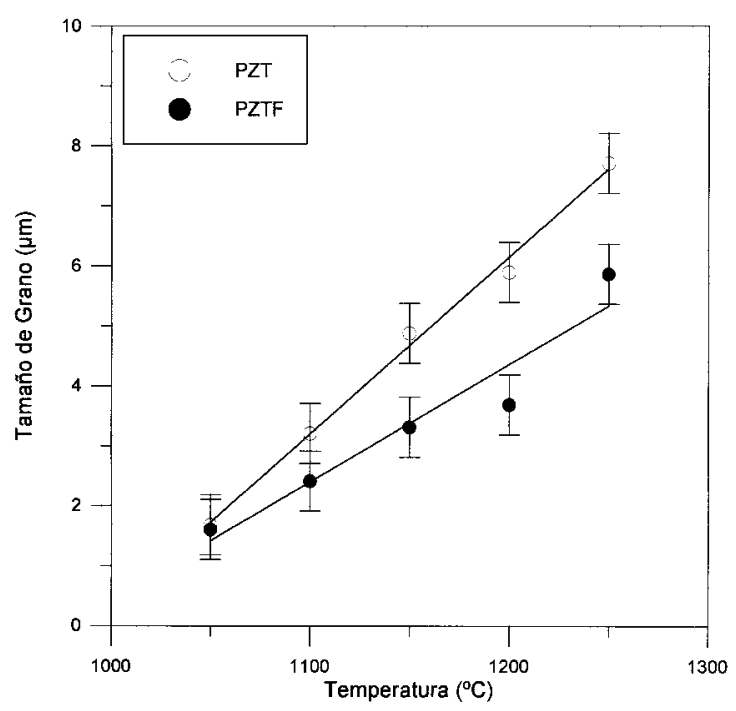

Figura 3. Tamaño de grano promedio en función de la temperatura de sinterización.
PZTF tendría una pérdida adicional de $0.06 \%$ y el material PZT de $0.23 \%$.

La diferencia de pérdida de peso entre el material PZT y PZTF $(\sim 0.15 \%)$ podría atribuirse a que el $\mathrm{PbO}$ libre en el material PZTF no se libera a la atmósfera sino mas bien puede reaccionar con el fósforo para formar compuestos fosfatados ricos en $\mathrm{PbO}$. Si se observa el diagrama de fases del sistema binario PbO- $\mathrm{P}_{2} \mathrm{O}_{5}$ (19), para la cantidad de $0.016 \%$ en peso de $\mathrm{P}_{2} \mathrm{O}_{5}$ presente en el material PZTF, se podrían formar compuestos en el siguiente intervalo de solución sólida: $\left(\mathrm{Pb}_{3} \mathrm{P}_{2} \mathrm{O}_{8}\right)_{x}\left(\mathrm{~Pb}_{4} \mathrm{P}_{2} \mathrm{O}_{9}\right)_{1-\mathrm{x}^{\prime}}$ con $0<\mathrm{x}<1$, que equivaldría en peso a una cantidad $<0.12 \%$ y quedando un residuo de $\mathrm{PbO}>0.03 \%$ en peso, independientemente del valor de $\mathrm{x}$; o bien $\left(\mathrm{Pb}_{3} \mathrm{P}_{2} \mathrm{O}_{8}\right)_{\mathrm{x}}\left(\mathrm{Pb}_{5} \mathrm{P}_{4} \mathrm{O}_{15}\right)_{1-\mathrm{x}}$, con $\mathrm{x}=0.12$, que constituye el $0.15 \%$ en peso. La formación de este último compuesto coincide con la reducción de un $0.15 \%$ de pérdida de peso del material PZTF con respecto al PZT. La formación de diferentes compuestos fosfatados ricos en plomo en este sistema es objeto de un estudio en desarrollo.

El análisis de la microestructura (figura 2) revela que las muestras de PZTF exhiben un tamaño de grano más pequeño y una distribución de granos más homogénea que las de PZT. No se ha observado la presencia de fases secundarias.

En la figura 3 se muestra la evolución del tamaño de grano para diferentes temperaturas de sinterización $\left(1050^{\circ} \mathrm{C}-\right.$ $1250^{\circ} \mathrm{C}$ ). Se aprecia que a $1050^{\circ} \mathrm{C}$ el tamaño de grano promedio de ambos materiales cerámicos son similares. A partir de esta temperatura $\left(1050^{\circ} \mathrm{C}\right)$, las curvas de ajuste lineal de las dos distribuciones de tamaño de grano se separan apreciablemente, siendo la del material PZTF la de menor pendiente. Este resultado indica la presencia de un mecanismo de control del tamaño de grano.

La figura 4 exhibe las distribuciones de tamaño de grano (ajuste polinómico) de los dos materiales cerámicos PZT y PZTF, a la temperatura de sinterización de $1200^{\circ} \mathrm{C}$. Los dos materiales presentan una distribución aproximadamente similar de tamaños de grano hasta valores de $\sim 4 \mu \mathrm{m}$. A partir de este tamaño de grano las dos curvas de distribución se diferencian notablemente. La curva de distribución de tamaño de grano del material PZTF se va cerrando rápidamente hasta

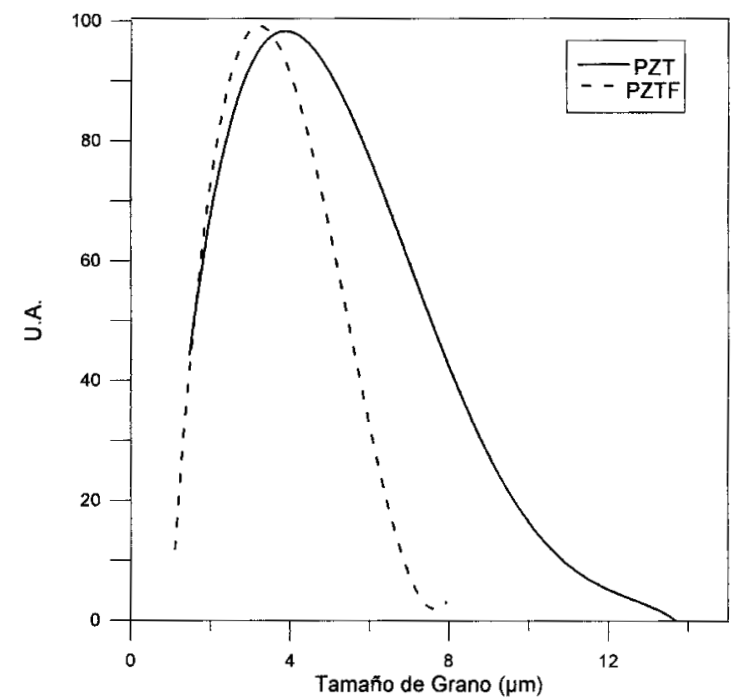

Figura 4. Distribución de tamaño de grano para los materiales cerámicos sinterizados a $1200^{\circ} \mathrm{C} / 2 \mathrm{~h}$. 
TABLA I. DisTRIBUCiÓN DE TAMAÑO DE GRANO DE PZT Y PZTF SINTERIZADOS A $1200^{\circ} \mathrm{C} / 2 \mathrm{H}$.

\begin{tabular}{|c|c|c|c|c|c|}
\hline Material & $\begin{array}{c}\text { TG medio } \\
(\mu \mathrm{m})\end{array}$ & $\begin{array}{c}\text { Desviación } \\
\text { Estándar } \\
(\mu \mathrm{m})\end{array}$ & $\begin{array}{c}\text { Tamaño } \\
\text { de Paso } \\
(\mu \mathrm{m})\end{array}$ & $\begin{array}{c}\text { Campo } \\
\text { Total } \\
(\mu \mathrm{m})\end{array}$ & $\begin{array}{c}\mathbf{N}^{\circ} \text { granos } \\
\text { contados }\end{array}$ \\
\hline PZT & 5.9 & 2.7 & 0.8 & $1.3-15.1$ & 423 \\
\hline PZTF & 3.7 & 1.4 & 0.5 & $0.9-8.2$ & 458 \\
\hline
\end{tabular}

anularse a $8 \mu \mathrm{m}$. Por el contrario, la curva de distribución del material PZT se extiende hasta $\sim 14 \mu \mathrm{m}$. En la tabla I se indican las características de obtención de estas dos curvas de distribución de tamaño de grano (figura 4) a la temperatura de sinterización de $1200^{\circ} \mathrm{C}$. Se observa la diferencia de la desviación estándar de ambas curvas de distribución, de 2.7 para el material PZT y 1.4 para el material PZTF. Estos resultados indican que durante el proceso de sinterización está actuando un mecanismo de control del tamaño de grano en el material PZTF, que permite que el crecimiento de los granos se retrase, obteniéndose tamaños de granos moderados con respecto a los del material PZT, para el mismo tiempo de sinterización. Además, la curva de distribución del material PZTF es más simétrica y presenta una desviación estándar menor que la del PZT, indicando una microestructura más homogénea del material, y en consecuencia, permitiendo obtener una efectiva reducción de las variaciones de las propiedades del material cerámico PZT.

El control del tamaño de grano que se observa en el material PZTF, así como su elevada densidad para una temperatura de sinterización inferior a la que se requiere para el material sin dopar, son resultados similares a los que se obtienen para el $\mathrm{BaTiO}_{3}$ dopado con fósforo (10). El diagrama de fases del sistema $\mathrm{PbO}-\mathrm{P}_{2} \mathrm{O}_{5}(19)$ recoge la formación de compuestos ricos en $\mathrm{PbO}$ y la formación de líquido a bajas temperaturas. De forma análoga a lo que se ha observado en el caso del $\mathrm{BaTiO}_{3}$ el recubrimiento de fósforo puede facilitar la formación de una fase reactiva rica en $\mathrm{PbO}$ que rodea las partículas de PZT en la primera etapa de la sinterización. Este recubrimiento ejerce una doble función facilitando la coalescencia de la porosidad y retrasando la cinética de los procesos de difusión que propician el crecimiento cristalino. El hecho de que las pérdidas de peso asociadas a la volatilización de $\mathrm{PbO}$ disminuyan en el material PZTF respecto al PZT (figura 2), parece apoyar esta hipótesis, ya que parte del $\mathrm{PbO}$ permanecería en el material formando un compuesto que contiene fósforo (11-12). Sin embargo, la existencia de fases secundarias no ha sido constatada por los procedimientos experimentales empleados. El bajo nivel de dopado unido a la posibilidad de incorporación en solución sólida al PZT del fósforo como donor eléctrico, dificultan dicha determinación. Un estudio más detallado sobre el mecanismo de incorporación del fósforo al PZT se está realizando en la actualidad.

En la tabla II se recogen las propiedades microestructurales, dieléctricas y piezoeléctricas de muestras de PZT y PZTF a la temperatura de mayor densificación $\left(1150^{\circ} \mathrm{C} / 2 \mathrm{~h}\right)$. Se puede apreciar que las propiedades dieléctricas y piezoeléctricas permanecen similares como resultado de la pequeña cantidad de fósforo añadido al PZT, $0.016 \%$ en peso. Sin embargo la constante dieléctrica $\mathrm{K}_{3}{ }^{\mathrm{T}}$ disminuye en el material PZTF debido a la probable formación de fases secundarias entre el $\mathrm{PbO}$ y el
TABLA II. PROPIEDADES MICROESTRUCTURALES, DIELÉCTRICAS Y PIEZOELÉCTRICAS DE PZT Y PZTF A LA TEMPERATURA DE SINTERIZACIÓN DE $1150^{\circ} \mathrm{C} / 2 \mathrm{H}$.

\begin{tabular}{|c|c|c|c|c|c|c|c|c|}
\hline Materiales & $\begin{array}{c}\text { Densidad } \\
\left(\mathrm{g} / \mathrm{cm}^{3}\right)\end{array}$ & $\begin{array}{c}\text { Pérdida } \\
\text { de Peso } \\
(\%)\end{array}$ & $\begin{array}{c}\text { Tamaño } \\
\text { Grano } \\
(\mu \mathrm{m})\end{array}$ & $\mathrm{K}_{3}{ }^{\top}$ & $\begin{array}{c}\tan \delta \\
(\%)\end{array}$ & $\mathrm{k}_{\mathrm{p}}$ & $\mathrm{k}_{\mathrm{t}}$ & $\begin{array}{c}\mathrm{d}_{33} \\
(\mathrm{pC} / \mathrm{N})\end{array}$ \\
\hline PZT & 7.72 & 0.83 & 4.9 & 1450 & 1.46 & 0.58 & 0.45 & 368 \\
\hline PZTF & 7.68 & 0.68 & 3.3 & 1306 & 1.42 & 0.63 & 0.46 & 358 \\
\hline
\end{tabular}

fósforo, constituyéndose un sistema de capacitores en serie con el material PZT original. El aumento de las constantes de acoplamiento electromecánico en el material PZTF podría ser resultado del incremento de tensiones internas en el material debido a la presencia de segundas fases en el borde de grano. Esto puede alterar la configuración de dominios, aumentando la densidad de paredes de los mismos (20-21) y por tanto una respuesta piezoeléctrica que incrementaría la conversión energética electromecánica. Además, algunos fosfatos de plomo tienen un comportamiento de tipo ferroelástico (22), que podría adicionalmente incrementar la conversión energética del material cerámico PZTF.

\section{CONCLUSIONES}

La modificación superficial de polvo cerámico PZT con ester fosfato produce una notable inhibición del crecimiento de grano que da lugar a una microestructura más homogénea. El polvo cerámico modificado con fósforo presenta una mayor rapidez de densificación alcanzando valores altos de densidad $\left(\sim 94 \% \delta_{t}\right)$ a temperaturas inferiores de sinterización. Las propiedades piezoeléctricas del material se mantienen en valores elevados. Se observa una reducción de las pérdidas de peso por volatilización del $\mathrm{PbO}$, posiblemente asociada a la reacción del $\mathrm{PbO}$ con el fósforo del material que puede dar lugar a la formación de compuestos fosfatados ricos en $\mathrm{PbO}$.

\section{AGRADECIMIENTOS}

Los autores agradecen a CICYT la financiación del presente trabajo dentro del proyecto MAT97-0694-C02-01 y al programa CYTED. D. Luis Alberto Celi agradece al programa de becas ICI de la AECI y a FUNDACYT de Ecuador por la ayuda brindada a través de una beca pre-doctoral, así como a la Escuela Politécnica Nacional, de Quito-Ecuador, por su auspicio.

\section{BIBLIOGRAFÍA}

1. J. F. Fernández, P. Durán, C. Moure, "Materiales cerámicos ferroeléctricos y sus aplicaciones". Bol. Soc. Esp. Ceram.y Vidrio, 32 [1], 5-15, (1993).

2. G. H. Haertling, "Ferroelectric Ceramics: History and Technology". J. Am. Ceram. Soc. 82 [4] 797-818 (1999).

3. W. E. Lee, W. M. Rainforth, “Ceramic Microstructures Property Control by Processing". pp. 67-121. Edit. Chapman and Hall, $1^{\text {a }}$ ed., Cambridge, Great Britain, 1994.

4. I. K. Lloyd, M. Kahn y S. Lang, "Effect of microstructural heterogeneities on piezoelectric behaviour of PZT ceramics". En Ceramic Transaction Vol.8, Dielectric Ceramic: Composition, Processing and Porperties, ed. H. C. Ling y M. Yan. The American Ceramic Society, Westerville, OH, 390-398, 1990.

5. K. Okazaki, K. Nagata, "Effects Of Density And Grain Size On The Elastic 
And Piezoelectric Properties $\mathrm{Of} \mathrm{Pb}(\mathrm{Zr}-\mathrm{Ti}) \mathrm{O}_{3}$ Ceramics".J. Soc. Mater. Sci. Jpn., 4, 404 (1972).

6. A. H. Webster, T.B. Weston, "The grain-size dependence of the electromechanical properties in lead zirconate-titanate ceramics". Can. Ceram. Soc. J., Vol 37, 51-54 (1968).

7. M. Kiyohara, K. Katoh, T. Mochizuki, S. Nagano, “Grain Size Dependence of Piezoelectricity and Actuator Characteristics for Piezoelectric Ceramics".J. Am. Cera. Soc. Jap., Vol 102, 548-553 (1994).

8. Clive Randall, Namchul Kim, John-Paul Kucera, Wenwu Cao, and Thomas Shrout, "Intrinsic and Extrinsic Size Effects in Fine-Grained MorphotropicPhase-Boundary Lead Zirconate Titanate Ceramics". J. Am. Ceram. Soc., 81[3] 677-88 (1998).

9. J. F. Fernández, A. C. Caballero, P. Durán, C. Moure, “Improving sintering behaviour of BaTiO3 by small doping additions".J. Mater. Sci. 31, 975-981 (1996).

10. A. C. Caballero, J. F. Fernández, C. Moure, P. Durán, “Effect Of Residual Phosphorus Left By Phosphate Ester On $\mathrm{BaTiO}_{3}$ Ceramics". Mater. Res. Bull. 32 (2) 221-229 (1997).

11. A. C. Caballero, J. F. Fernández, C. Moure, P. Durán, "Phase formation in phosphorus doped $\mathrm{BaTiO}_{3}$ ". Mater. Letters 35, $72-77$ (1998).

12. A. C. Caballero, M. Villegas, J. F. Fernández, C. Moure, P. Durán, P. Florian, J. Coutures, "Reactive Sintering of Phosphorous Coated $\mathrm{BaTiO}_{3}$ ". J. Eur. Ceram. Soc. 19, 979-983 (1999).

13. L. A. Celi, A. C. Caballero, M. Villegas, P. Durán, C. Moure, J. F. Fernández, "Efecto de las características de los polvos cerámicos sobre la densificación y propiedades de materiales cerámicos $\mathrm{PZT}^{\prime}$. Bol. Soc. Esp. Ceram.Vidrio, 38 [5] 493-497 (1999)
14. T.Shrout, P.Papet, S.Kim, G.Lee, "Conventionally Prepared Submicrometer Lead-Based Perovskite Powders by Reactive Calcination". J. Am. Ceram. Soc.73[7]1862-67(1990).

15. F. F. Lange, "Sinterability of Agglomerated Powders". J. Am. Ceram. Soc. 67 [2] 83-89 (1984).

16. J. S. Reed, T. Carbone, C. Scott, S. Lukasiewics, "Some Effects of Aggregates and Agglomerates in the Fabrication of Fine Grained Ceramics"; pp. 171-80 en Processing of Crystalline Ceramics. Editado por Palmour III, R. F. Davis y T. M. Hare Plenum, New York,1978.

17. IEEE Standards on Piezoelectricity, ANSI/IEEE Std. 176. The institute of Electrical and Electronics Engineers, New York (USA) (1987).

18. R. B. Atkin, R. L. Holman, R. M. Fulrath, "Substitution of Bi and $\mathrm{Nb}$ Ions in Lead Zirconate-Titanate". J. Am. Ceram. Soc. 54[2] 113-115 (1971).

19. Ernest Levin, Carl Robbins, Howard McMurdie, "Phase Diagrams for Ceramists". Compilado en la National Bureau of Standards (EUA). Editor Margie Reser. The Am. Ceram. Soc., pp. 117 (1964).

20. Sang-Beom Kim, Tai-Joo Chung, Doh-Yeon Kim, "Effect of External Compressive Stress on the Domain Configuration of Barium Titanate Ceramics". J. Europ. Ceram. Soc. 12, 147-151 (1993).

21. P. Sarrazin, B. Thierry, J. C. Niepce, "Forming Pressure Dependence of the Ferroelectric Domain Structure in Green Barium Titanate Pellets". J. Europ. Ceram. Soc. 15, 623-629 (1995).

22. E. Salje, "Phase Transitions in Ferroelastic and Co-elastic Crystals". Pp. 1-5. Ed. Cambridge University Press, student edit. first, Great Britain (1993). 